\title{
Correction to: Spectral analysis of the diffusion operator with random jumps from the boundary
}

\section{Martin Kolb ${ }^{1}$ - David Krejčiř́ík ${ }^{2}$}

Published online: 19 August 2019

○ Springer-Verlag GmbH Germany, part of Springer Nature 2019

\section{Correction to: Math. Z. (2016) 284:877-900 https://doi.org/10.1007/s00209-016-1677-y}

\begin{abstract}
We correct a wrong statement in the original article that the studied operator is quasi-accretive. In fact, in this corrigendum we show that the numerical range of the operator coincides with the whole complex plane. We argue that the other statements in the original article still hold.
\end{abstract}

In the original article we studied the operator $H$ in $L^{2}\left(\left(-\frac{\pi}{2}, \frac{\pi}{2}\right)\right)$ defined by

$$
H \psi:=-\psi^{\prime \prime}, \quad \psi \in \mathrm{D}(H):=\left\{\psi \in H^{2}\left(\left(-\frac{\pi}{2}, \frac{\pi}{2}\right)\right) \mid \psi\left(-\frac{\pi}{2}\right)=\psi\left(\frac{\pi}{2} a\right)=\psi\left(\frac{\pi}{2}\right)\right\} .
$$

On page 880 in the original article we presented a proof that $H$ is quasi-accretive, but an additional derivative was missing on the second line of the proof, making the total argument irreparable. ${ }^{1}$ The correct identity reads

$$
\Re(\psi, H \psi)=\left\|\psi^{\prime}\right\|^{2}-\frac{1}{2} \int_{-\frac{\pi}{2}}^{\frac{\pi}{2}}|\psi|^{2^{\prime \prime}}(x) d x
$$

and it follows that the quasi-accretivity actually does not hold. In fact, the whole complex plane belongs to the numerical range.

Proposition $\{(\psi, H \psi) \mid \psi \in D(H),\|\psi\|=1\}=\mathbb{C}$.

Proof ${ }^{2}$ We employ the identity

$$
(\psi, H \psi)=\left\|\psi^{\prime}\right\|^{2}-\bar{\psi}\left(\frac{\pi}{2}\right)\left[\psi^{\prime}\left(\frac{\pi}{2}\right)-\psi^{\prime}\left(-\frac{\pi}{2}\right)\right],
$$

1 We are grateful to Matěj Tušek for pointing out the mistake to us.

2 The present proof is based on an initial idea of Matěj Tušek.

The original article can be found online at https://doi.org/10.1007/s00209-016-1677-y.

$\bowtie \quad$ David Krejčiř́ik

david.krejcirik@fjfi.cvut.cz

1 Department of Mathematics, Universität Paderborn, Warburger Str. 100, 33098 Paderborn, Germany

2 Department of Mathematics, Faculty of Nuclear Sciences and Physical Engineering, Czech Technical University in Prague, Trojanova 13, 12000 Prague 2, Czech Republic 
which follows by integrating by parts and using the boundary conditions. Let $\phi \in C^{\infty}(\mathbb{R})$ be an arbitrary complex-valued function such that its support is contained in $\left(\frac{\pi}{2} a, \frac{\pi}{2}\right]$. We set

$$
\psi_{\varepsilon}(x):= \begin{cases}1+\sqrt{\varepsilon} \phi\left(\frac{\pi}{2}-\varepsilon^{-1}\left(\frac{\pi}{2}-x\right)\right) & \text { if } x \in\left[\frac{\pi}{2} b_{\varepsilon}, \frac{\pi}{2}\right] \\ 1 & \text { otherwise }\end{cases}
$$

with $b_{\varepsilon}:=1-\varepsilon(1-a)$ and any $\varepsilon \in(0,1]$. Clearly, $\psi_{\varepsilon} \in \mathrm{D}(H)$ and a straightforward calculation yields

$$
\begin{aligned}
\left(\psi_{\varepsilon}, H \psi_{\varepsilon}\right) & =\left\|\phi^{\prime}\right\|^{2}-\frac{\phi^{\prime}\left(\frac{\pi}{2}\right)}{\sqrt{\varepsilon}}, \\
\left\|\psi_{\varepsilon}\right\|^{2} & =\pi+2 \varepsilon^{3 / 2} \Re \int_{\mathbb{R}} \phi(y) d y+\varepsilon^{2} \int_{\mathbb{R}}|\phi(y)|^{2} d y \underset{\varepsilon \rightarrow 0}{\longrightarrow} \pi .
\end{aligned}
$$

Since $\phi^{\prime}\left(\frac{\pi}{2}\right)$ is an arbitrary complex number, the desired claim follows by sending $\varepsilon$ to zero and recalling the convexity of the numerical range.

As a consequence of the lack of quasi-accretivity, the operator $H$ is not quasi-m-accretive and the corresponding part of Proposition 3 in the original article does not hold. On the other hand, $H$ is still an operator with compact resolvent. To make the argument of the proof of Proposition 3 in the original article work, it is enough to show that the resolvent of $H$ exists for one complex $z$. However, given an arbitrary $F \in L^{2}\left(\left(-\frac{\pi}{2}, \frac{\pi}{2}\right)\right)$, the equation $(H-z) \psi=F$ admits an explicit solution for every $z \notin \sigma_{\mathrm{p}}(H)$ (easily obtained by the variation of constants, for example). Alternatively, one can use the explicit formula for the resolvent kernel given in Proposition 5 in the original article (the statement remains valid for all $\left.\lambda \in \mathbb{C} \backslash\left[\sigma_{\mathrm{p}}(H) \cup \sigma_{\mathrm{p}}\left(H^{0}\right)\right]\right)$.

The quasi-m-accretivity of $H$ was also used in Theorem 4 in the original article claiming that the eigenfunctions of $H$ together with the generalised eigenfunctions form a complete set in $L^{2}\left(\left(-\frac{\pi}{2}, \frac{\pi}{2}\right)\right)$. This statement still holds. Indeed, instead of using the sufficient condition [2, Thm. VII.8.1] based on the quasi-m-accretivity (which is not available), one can use a more robust result of Dunford and Schwartz [1, Corol. XI.6.31 $]^{3}$ requiring that the resolvent is a Hilbert-Schmidt operator and admits an algebraic decay with respect to the spectral parameter along several rays. These properties can be easily checked by using the explicit formula for the resolvent kernel given in Proposition 5 in the original article. Analogously, the eigenfunctions of the adjoint $H^{*}$ together with the generalised eigenfunctions form a complete set in $L^{2}\left(\left(-\frac{\pi}{2}, \frac{\pi}{2}\right)\right)$, making the conclusion below Corollary 1 in the original article still valid (although $H^{*}$ is not quasi-accretive either).

In the remaining parts of the original article the (unavailable) quasi-accretivity of $H$ was not used and the arguments and results remain valid. Summing up, all the theorems, propositions, lemmata and corollaries presented throughout the paper in the original article remain valid, with the unique exception of Proposition 3 where "quasi-m-accretive" should be replaced with "closed". In particular, the three main theorems presented in the introduction in the original article remain valid without any changes.

Acknowledgements We are grateful to our colleagues Lyonell Boulton,Vladimir Lotoreichik, Konstantin Pankrashkin and Matěj Tušek for useful discussions. The research of D.K. was partially supported by the GACR Grant no. 18-08835S.

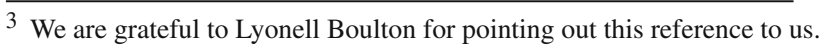




\section{References}

1. Dunford, N., Schwartz, J.T.: Linear operators, part 2, spectral theory. Wiley-Interscience, Hoboken (1963)

2. Gohberg, I.C., Goldberg, S., Kaashoek, M.A.: Classes of linear operators, vol. 1. Birkhäuser, Basel (1990)

Publisher's Note Springer Nature remains neutral with regard to jurisdictional claims in published maps and institutional affiliations. 